\title{
PROTESTE ED EMANCIPAZIONE ALLA FRONTIERA EUROPEA. IL CASO DI LAMPEDUSA
}

\author{
Protests and emancipation at the European border. \\ The case of Lampedusa
}

\begin{abstract}
Annalisa Lendaro ${ }^{1}$
Riassunto. Questo articolo si propone di approfondire a livello teorico i legami tra proteste ed emancipazione dei migranti senza status legale, che si trovano in situazione di detenzione amministrativa sull'isola italiana di Lampedusa. II presente contributo mette in evidenza il fatto che l'agency dei migranti, seppur privi di libertà e di uno status legale, è determinante per sbloccare una situazione di crisi in un contesto simile allo stato di eccezione descritto da Agamben. Più precisamente, la protesta dei migranti di Lampedusa mostra in che modo la sospensione dello stato di diritto apra degli spiragli per, non solo denunciare l'ingiustizia di alcune norme, ma anche per agire.

Parole chiave: detenzione amministrativa, movimenti di protesta, stato di eccezione, emancipazione dei migranti.

Abstract. This paper provides a theoretical analysis of the links between the protests organized by migrants without legal status and their emancipation, while they are officially detained in a closed center on the Italian island of Lampedusa. This study shows that the agency of these migrants is fundamental to the issue of this crisis, within a context similar to the Agamben' state of exception. More precisely, the protest in Lampedusa highlights the way in which the adjournement of the rule of law can open up new opportunities to, not only denounce the injustice of some norms, but also to act against them.
\end{abstract}

Keywords: administrative detention, protest movements, state of exception, empowerment of migrants.

1 Ricercatrice al CNRS, Centre National de la Recherche Scientifique, CERTOP. Toulouse, France. 


\section{Introduzione}

Da quindici anni a questa parte, le strutture per la detenzione amministrativa dei migranti si moltiplicano sul suolo europeo, in particolare nelle zone frontaliere ${ }^{2}$. Se in origine questi dispositivi hanno fatto parte di un sistema improvvisato di accoglienza dei migranti e richiedenti asilo, oggi sono totalmente istituzionalizzati e rappresentano uno dei pilastri delle politiche migratorie europee ${ }^{3}$.

Questi campi ${ }^{4}$ adempiono alla funzione principale di selezione dei migranti, e di distinzione tra accettabili e indesiderati. A seconda del grado di "desiderabilità" 5 , la frontiera risulta loro più o meno permeabile.

Quella che i media europei chiamano la "crisi dei rifugiati" ha messo in luce una gerarchia delle nazionalità che permettono ai richiedenti asilo di ottenere protezione: oramai, solo i cittadini siriani e irakeni hanno speranze concrete di penetrare nella "fortezza Europa", mentre i cittadini di altri paesi lacerati da conflitti o governati da dittature spietate, come l'Afghanistan, il Pakistan, o la Somalia, si ritrovano spesso bloccati alle frontiere esterne dell'UE, vittime del filtraggio operato da paesi limitrofi come la Macedonia o la Serbia.

La lista dei paesi di origine considerati "sicuri" (safe countries) sembra inoltre variare a seconda della volontà più o meno forte dei paesi dell'UE di adempiere al loro dovere di accoglienza iscritto nella Convenzione di Ginevra. Questa lista funge da variabile di aggiustamento: se da un lato l'UE ha visto il numero di rifugiati siriani ed irakeni aumentare esponenzialmente nel 2015 e nel 2016, dall'altro, ha rifiutato quasi sistematicamente I'ingresso ai cittadini kossovari, ukraini, ecc., i cui paesi di origine sono considerati sufficientemente sicuri.

Sempre più spesso alle frontiere esterne dell'Europa i centri di identificazione per migranti sono sovraffollati e offrono condizioni di permanenza indecorose ${ }^{6}$. Inoltre, poiché la regolamentazione della detenzione amministrativa non è ufficializzata in nessun testo comune agli stati membri dell'UE, la loro permanenza in questi centri può durare diversi mesi, senza che una decisione di giustizia sia stata presa davanti ad un giudice che li abbia riconosciuti colpevoli di un qualche crimine. E di conseguenza, senza possibilità di appello.

In questo contesto, il presente contributo analizza il caso di una protesta di migranti in situazione di detenzione amministrativa sull'isola di Lampedusa

\footnotetext{
2 Cf. www.en.closethecamps.org; TASSIN, Louise. Le mirage des hotspots. Nouveaux concepts et vieilles recettes à Lesbos et Lampedusa.

3 CAMPESI, Giuseppe. La detenzione amministrativa degli stranieri.

4 AGIER, Michel. Un monde de camps.

5 AGIER, Michel. Managing the undesirables: refugee camps and humanitarian government.

6 OPEN ACCES NOW. La face cachée des camps d'étranger-e-s en Europe.
} 
(Italia) che ha avuto luogo nel luglio 2013. Questa protesta non è per nulla un caso isolato e specifico alla piccola isola italiana: recentemente, delle rivolte simili si sono prodotte sulle isole di Kos e Lesbos, in Grecia, o ancora a Idomeni, alla frontiera greco-macedone.

L'articolo si concentra sulla portata emancipatrice di una protesta che, non solo ha preso forma al di fuori del sistema di reclusione, poiché i migranti hanno manifestato per le strade, occupando la piazza principale dell'isola, ma ha inoltre avuto come parola d'ordine il non rispetto delle norme in vigore riguardandi la schedatura e l'identificazione dei migranti, considerate ingiuste perché lesive del diritto alla mobilità di ciascun essere umano.

Partendo da questo esempio, il presente contributo esamina il rapporto tra la violazione della legge e dei diritti dei migranti su un'isola-frontiera e l'emergere di rivolte emancipatrici. In particolare, il testo approfondisce la questione dell'agency dei migranti in un contesto simile a quello dello stato di eccezione ${ }^{7}$.

Il caso di Lampedusa si presta quindi a una riflessione più generale sulle condizioni per la nascita di un processo di emancipazione ${ }^{8}$, inteso come un percorso di presa di coscienza e di liberazione collettiva da un sistema oppressivo, durante il quale un gruppo si sottrae a una situazione subalterna e ottiene, eventualmente, il riconoscimento di diritti o del diritto ad avere diritti ${ }^{9}$.

\section{Lampedusa: l'eccezione che diventa la regola}

L'Italia e la Grecia sono attualmente le due principali porte d'ingresso dell'Europa, soprattutto da quando le partenze si sono intensificate ad est del bacino mediterraneo (crisi siriana, guerra in Afghanistan, El in Irak). Ma se Lampedusa è stata l'emblema dell'immigrazione irregolare fino a qualche anno fa, l'isola ha recentemente ceduto il suo podio mediatico alle greche Kos e Lesbos. Oggi poco si legge o si vede di Lampedusa nei media.

Eppure I'isola continua ad essere una destinazione ambita per coloro i quali tentano la traversata del Mediterraneo partendo su imbarcazioni di fortuna dalla Libia, dalla Tunisia o dall'Egitto. In particolare, per i migranti che fuggono dal Corno d'Africa (Somalia, Eritrea, Etiopia) o dall'Africa subsahariana (Niger, Mali, Gambia, Guinea, Costa d'Avorio ecc.) Lampedusa, assieme a Malta, rappresenta il primo territorio europeo verso cui dirigersi.

Ma per molti di loro questa piccola isola italiana di soli 20 chilometri quadrati, abitata da sole 6000 persone, è solamente una tappa verso l'Europa continentale. Spesso infatti la destinazione finale dei migranti che arrivano a

\footnotetext{
7 AGAMBEN, Giorgio. Lo stato di eccezione.

8 LACLAU, Ernesto. Emancipazione/i.

9 ARENDT, Hannah. The origins of totalitarianism.
} 
Lampedusa è piuttosto un paese del nord Europa (Svezia, Germania, ecc.), dove alcuni hanno già contatti, amici o parenti ad aspettarli.

Lampedusa, nella sua esiguità spaziale, possiede uno dei centri di primo soccorso e accoglienza (CPSA) più frequantati del paese. La decisione di costruire un CPSA alla fine degli anni 1990 ha progressivamente trasformato il suo tessuto sociale. Nel giro di 20 anni, Lampedusa è diventata un avamposto militare e un'antenna fondamentale per il controllo e la protezione della frontiera sud dell' $U E^{10}$, provocando non solo il trasferimento sistematico dei migranti intercettati in mare, ma anche e soprattutto l'arrivo di personale militare e di operatori del settore umanitario.

Questo ruolo specifico di "guardiana della frontiera" si è sviluppato assieme ad una retorica dell'urgenza, periodicamente utilizzata (e strumentalizzata) dalla classe politica non solo italiana, ma anche europea. Le immagini dei barconi e del centro strapieno di migranti hanno fatto il giro del mondo. Questa mediatizzazione intensiva della presenza dei migranti a Lampedusa è possibile grazie ad un sistema di accoglienza che funziona da sempre sulla violazione della legge e dei diritti dei migranti.

II CPSA è stato concepito come centro di primo soccorso, e la sua funzione legale è quindi quella di fornire le prime cure ai migranti che sopravvivono alla traversata del Mediterraneo, e di prepararne il trasferimento verso un altro centro idoneo ad accoglierli per periodi più lunghi. Invece i migranti sono regolarmente trattenuti ben oltre le 72 ore previste $^{11}$, e si ritrovano rinchiusi a volte per mesi in un centro che non è adatto ad accogliere uomini, donne e bambini in situazione di promiscuità, che non offre gli spazi necessari per una permanenza prolungata (assenza di stanze attrezzate per i pasti), e soprattutto che non possiede sufficienti posti letto (circa 380, a fronte di più di mille persone accolte durante i mesi estivi).

Lo stato di diritto è sospeso: i migranti trattenuti in situazione di detenzione amministrativa sono privi di uno status legale (non sono ancora dei richiedenti asilo poiché non hanno ancora effettuato la richiesta ufficiale di protezione), e non hanno la possibilità di far ricorso alla giustizia italiana. A fronte di questi elementi, le tensioni sono ricorrenti e poiché il centro è spesso sovraffollato, le forze dell'ordine permettono ai migranti di uscire dal CPSA al fine di evitare

${ }^{10}$ CUTTITTA, Paolo. Lo spettacolo della frontiera.

11 Il decreto interministeriale del 16 febbraio 2006 definisce il centro per stranieri di Lampedusa come un Centro di Primo Soccorso e di Accoglienza (CPSA). Dal punto di vista giuridico, la sua missione è quella di garantire ai migranti le prime cure, prima di poterli trasferire verso un Centro di Accoglienza per Richiedenti Asilo (CARA) oppure un Centro di Identificazione ed Espulsione (CIE). Tuttavia, il decreto non precisa le condizioni e le modalità specifiche dell'accoglienza temporanea nei CPSA. L'unica nota riguardante la durata della permanenza prevede che la detenzione non superi il tempo necessario alle prime attività di soccorso e di cura, stimate a massimo 72 ore. 
epidemie e soprattutto rivolte. In questo contesto d'ordinaria eccezione, Lampedusa diventa uno spazio di protesta e di resistenza.

\section{La protesta}

La manifestazione dei migranti del 2013 ha preso forma in un clima molto teso. A luglio di quell'anno, più di 1000 persone sono rinchiuse nel CPSA da diverse settimane. Inoltre, gli arrivi di nuovi migranti continuano senza sosta, preoccupando le autorità. Le forze dell'ordine, temendo una possibile sommossa, decidono di lasciar uscire i migranti dal centro. Tuttavia, I'ostilità di una parte degli isolani è palpabile, soprattutto da parte di coloro i quali vivono di un'attività legata al turismo: i migranti liberi per l'isola sono visti come un problema per lo sviluppo delle attività turistiche e come un deterrente per i visitatori.

Nonostante ciò, il 20 luglio un gruppo di circa 200 migranti, per lo più Eritrei e guidati da un gruppo di giovani in possesso di titoli di studio, decide di manifestare pacificamente, organizzando una marcia spontanea di protesta che tocca i punti nevralgici dell'isola, e che finisce con l'occupazione della piazza principale, davanti alla chiesa. I manifestanti, esasperati dall'attesa e dalle condizione indecorose di accoglienza nel centro, rivendicano il diritto di partire da Lampedusa senza essere schedati, senza quindi registrare le proprie impronte digitali. II regolamento europeo chiamato Dublino II (diventato Dublino III nel 2014), vieta ai migranti di spostarsi in un altro stato dell'UE per richiedere l'asilo o la protezione umanitaria. Di conseguenza, lasciare le proprie impronte a Lampedusa significherebbe per loro essere obbligati a richiedere I'asilo in Italia, quando questo paese non è la loro meta.

Una soluzione a questa crisi viene trovata l'indomani, al termine di una riunione informale tenutasi tra il sindaco, i rappresentanti delle forze dell'ordine, della chiesa e del Ministero dell'Interno. Al fine di evitare gli scandali e le polemiche, locali e internazionali, i partecipanti alla riunione decidono che un certo numero di migranti originari del Corno d'Africa potranno essere trasferiti verso I'Italia continentale senza essere fotosegnalati.

Non è quindi sul campo legale che la partita è stata giocata. A differenza delle proteste e degli scioperi condotti dai sans papiers in Francia ${ }^{12}$ o dai sinpapeles in Spagna ${ }^{13}$, i manifestanti di Lampedusa non hanno potuto contare su degli esperti militanti capaci di difendere la loro causa e tradurre le loro rivendicazioni nell'arena politica e giudiziaria ${ }^{14}$. Questo non solo perché a Lampedusa, territorio ultraperiferico, mancano le rappresentanze sindacali e le organizzazioni di difesa dei diritti dei migranti, ma soprattutto perché i

\footnotetext{
${ }^{12}$ BARRON, Pierre et alii. On bosse ici, on reste ici!: La grève des sans-papiers: une aventure inédite.

${ }^{13}$ BARBERO, Iker. Expanding acts of citizenship: the struggles of Sinpapeles Migrants.

${ }^{14}$ ISRAËL, Liora. Usages militants du droit dans I'arène judiciaire: le cause lawyering.
} 
manifestanti rivendicano il diritto di violare la legge (i.e. I'obbligo di registrare le loro impronte) e non il suo rispetto. Il loro claim vuole mettere in evidenza I'ingiustizia di una legge che li priva della libertà di movimento.

\section{Stato d'eccezione ed agency dei migranti}

Vasta è la produzione accademica dedicata ai movimenti sociali dei migranti e alle loro proteste ${ }^{15}$. In questi studi il migrante che protesta si rivolge al governo del suo paese di residenza e ai suoi cittadini per chiedere la ridefinizione dei criteri di appartenenza alla società e per rivendicare più diritti (al lavoro, alla casa, alla salute ecc.). La figura che ne emerge è quella di un migrante intraprendente, cosciente dei propri diritti, capace di formulare le proprie richieste indirizzandosi a coloro i quali hanno facoltà di decidere in materia di res publica.

Al polo opposto, quello della letteratura sullo stato d'eccezione, il soggetto viene invece presentato come completamente schiacciato dalla forza del potere sovrano. Il migrante è allora visto come vittima di un sistema che non gli attribuisce alcuno spazio, nessun diritto, e che lo riduce alla vita nuda (bare life $)^{16}$.

Il caso di Lampedusa merita un approfondimento: i migranti che hanno manifestato nel luglio 2013 sulla piccola isola italiana hanno agito in un contesto particolarmente ostile, caratterizzato dalla mancanza di appoggi esterni, dalla reclusione amministrativa prolungata, dall'isolamento. Nonostante i manifestanti fossero privi di status legale, e nonostante lo stato di eccezione vigente sull'isola li abbia relegati in uno spazio al di fuori dello stato di diritto, privati della possibilità di ricorrere alla giustizia, questi migranti hanno saputo far pressione per ottenere la violazione di una norma considerata ingiusta. Questi migranti non sono quindi completamente privi di risorse, anzi: la protesta costituisce un momento importante del processo di presa di coscienza collettivo che permette all'individuo di costituirsi in quanto essere politico (being political) $)^{17}$.

\footnotetext{
${ }^{15}$ Solamente sull'Europa: ANDERSON, Bridget. Mobilizing migrants, making citizens: migrant domestic workers as political agents; KOOPMANS, Ruud (ed.). Contested citizenship: Immigration and cultural diversity in Europe; GIUGNI, Marco, PASSY, Florence. Migrant mobilization between political institutions and citizenship regimes: A comparison of France and Switzerland; SOYSAL, Yasemin Nuhoğlu. Changing parameters of citizenship and claims-making: Organized Islam in European public spheres; MCGREGOR, JoAnn. Contestations and consequences of deportability: hunger strikes and the political agency of non-citizens; OLIVERI, Federico. Migrants as activist citizens in Italy: understanding the new cycle of struggles; ELLERMANN, Antje. Undocumented migrants and Resistance in the Liberal State.

${ }^{16}$ AGAMBEN, Giorgio. Homo sacer: Sovereignty and bare life; RAJARAM, Prem Kumar, GRUNDYWARR, Carl. The irregular migrant as homo sacer: Migration and detention in Australia, Malaysia, and Thailand.

17 ISIN, Engin Fahri. Being political: genealogies of citizenship.
} 


\section{Lo stato d'eccezione alle frontiere dell'Europa: vie di fuga}

Il concetto di Stato di eccezione è utilizzato dal filosofo italiano Giorgio Agamben ${ }^{18}$ per evocare la sospensione dello stato di diritto. Questa sospensione è la conseguenza della decisione del potere sovrano di mantenere alcuni individui all'esterno delle frontiere della polis o della cité, in uno spazio in cui la protezione fisica e giuridica degli individui è negata. Agamben ricorre all'esempio dell'homo sacer dell'Impero Romano per spiegare in che modo questo Stato di eccezione influisce sulla vita degli individui. L'homo sacer è colui che può essere ucciso ma non sacrificato: questo individuo è privato di ogni diritto e in particolare di quello di essere protetto dalla legge. Di conseguenza, qualsiasi altra persona può togliergli la vita senza essere punito. Lo stato di eccezione è quindi rappresentato da tutte le situazioni che concorrono a estendere il potere del sovrano (lo Stato), come per esempio la sua facoltà di confinare alcuni individui o gruppi al di fuori dello stato di diritto, privandoli di qualsiasi riconoscimento e protezione giuridica, fino al punto di mettere in pericolo la loro vita. Un esempio di Stato di eccezione utilizzato dal filosofo è quello del campo, uno spazio chiuso in cui vengono imprigionate persone che non sono state giudicate colpevoli di alcun crimine. Qui, la legge è sospesa e l'individuo privo di diritti è ridotto alla semplice vita biologica, alla vita nuda (bare life).

Lampedusa è un esempio pertinente per mettere in discussione la figura del migrante come vittima impotente dello Stato di eccezione. A Lampedusa, la reclusione e la deportazione sono delle esperienze che traducono lo Stato di eccezione nel concreto del percorso dei migranti.

Il campo, in questo caso il centro per migranti, garantisce loro la sperimentazione istituzionalizzata della violenza fisica, della privazione prolungata della libertà, del rischio di epidemie, dell'attesa della propria sorte in un contesto psicologico degradante e rischioso (promiscuità tra uomini e donne e tra adulti e bambini). Queste situazioni si presentano regolarmente in un contesto di ordinaria violazione della legge da parte delle stesse istituzioni pubbliche, senza possibilità per le vittime di far ricorso alla giustizia. Le possibilità di rivolta e di emancipazione si manifestano quindi, a fortiori, al di fuori della legge.

Il caso della protesta dei migranti a Lampedusa è un esempio di quello che Brophy ${ }^{19}$ descrive, in filosofia del diritto, come la breccia nello stato di eccezione: lo Stato che governa attraverso l'eccezione, crea le condizioni necessarie alla propria fallibilità. In effeti, i migranti rinchiusi nel centro sono confinati in uno spazio di esclusione, di esteriorità rispetto alla società e alle leggi che normalmente la reggono. Questa situazione conduce ad una protesta che avanza una richiesta di giustizia al di fuori ed al di là della legge in vigore.

\footnotetext{
18 AGAMBEN, Homo sacer..., op. cit.

${ }^{19}$ BROPHY Susan D. Lawless sovereignty: challenging the State of Exception.
} 
Come durante il regime coloniale descritto da Fanon ${ }^{20}$, i migranti sono considerati dallo Stato come dei soggetti d'eccezione, nel senso che sono trattati in quanto esseri umani che non hanno gli stessi diritti dei cittadini. Lo Stato o chi per lui può esercitare il suo potere decisionale su di loro, ma non garantisce la protezione dello stato di diritto alla quale possono appellarsi gli altri soggetti, i cittadini. I migranti sono in questo senso esteriorizzati ed inferiorizzati. Tuttavia, questa esteriorità assegnata può diventare una risorsa.

La visione di Agamben, che tende a ridurre i migranti rinchiusi nei campi a vittime impotenti, merita di essere discussa. Il caso della protesta di Lampedusa dimostra che, in un contesto simile a quello dello stato di eccezione agambiano, i migranti possono agire al di fuori della legge facendo pressione sui poteri pubblici, obbligandoli a fare dei compromessi. Più precisamente, questa protesta e i suoi risultati evidenziano il fatto che l'"eccezione produce eccezione": dopo settimane di attesa all'interno di un centro sovrappopolato, senza possibilità di fare appello alla giustizia, i migranti organizzano una marcia illegale, poiché la loro presenza nello spazio pubblico non è autorizzata ed implica di infrangere l'obbligo della reclusione. Oltretutto, la richiesta dei migranti è portatrice di un'altra eccezione: quella di lasciar partire i manifestanti senza che le loro impronte siano registrate dalle forze dell'ordine.

Questo caso è esemplare per lo studio delle condizioni necessarie affinché dei migranti privi di diritti e di uno status legale riescano a sovvertire, anche se solo temporaneamente, il sistema di accoglienza italiano ed europeo attraverso un'azione che rivendica la violazione di una legge considerata ingiusta.

L'esperienza della reclusione prolungata e la possibilità di uscire dal centro a causa del suo sovraffollamento è determinante per l'emancipazione di questo gruppo di migranti. L'emergere di una richiesta collettiva (lasciare I'isola senza essere fotosegnalati, per poter continuare il viaggio verso un altro paese europeo) è il segno della nascita di un soggetto politico, di un insieme di individui che si riconoscono come aventi dei diritti, o meglio, come aventi il diritto di avere dei diritti ${ }^{21}$. La manifestazione di Lampedusa, per nulla unica nel suo genere, permette ai migranti di esprimere un sentimento di ingiustizia, legato alla permanenza del centro in condizioni indecorose, certo, ma anche all'obbligo ingiusto di doversi fermare in Italia a chiedere l'asilo.

Il caso di questa manifestazione è un esempio di come dei migranti privi di status possano sperimentare delle forme di esistenza politica che rimettono in discussione la giustizia delle norme in vigore, e più in generale delle politiche migratorie contemporanee.

\footnotetext{
${ }^{20}$ FANON, Frantz. The wretched of the earth.

21 ARENDT, op. cit.
} 


\section{Conclusioni}

Molte sono le ricerche già pubblicate sullo stato di eccezione alle frontiere ${ }^{22}$. Quelle sull'agency dei migranti che si trovano in situazioni particolarmente critiche, dove lo stato di diritto è sospeso, sono meno numerose.

Questa ricerca ha voluto approfondire la questione delle condizioni per l'emergere di un processo di emancipazione di migranti senza status confrontati allo stato di eccezione tipico di un'isola frontiera e del dispositivo della reclusione amministrativa. In particolare, attraverso la protesta di Lampedusa i migranti sono riusciti a costituirsi in subjects of legality ${ }^{23}$, sperimentando una forma di being politica ${ }^{24}$ che passa per la rivendicazione del diritto alla mobilità. Nonostante la reclusione amministrativa e la condizione di privazione di diritti, questi migranti sono riusciti ad approfittare della stagione turistica e della presenza dei molti villeggianti per creare una situazione di apprensione per i potenziali disordini ${ }^{25}$. Tale apprensione ha condotto i decisori politici ad assecondare la loro richiesta.

Questa ricerca ha inoltre voluto dimostrare che la violazione della legge non è incompatibile con la nascita di un percorso di emancipazione. Questa riflessione, a differenza dalle analisi dicotomiche che distinguono il cittadino e lo straniero, propone di pensare la figura del 'cittadino senza status', legalmente privo di diritti e tuttavia protagonista di un processo di autoidentificazione come attore politico, capace di manifestare per criticare un sistema legale considerato ingiusto, quindi non degno di essere rispettato.

\section{Bibliografia}

AGAMBEN, Giorgio. Homo sacer: Sovereignty and bare life. Trans. D. Heller-Roazen. Stanford: Stanford University Press, 1998.

AGAMBEN, Giorgio. Lo stato di eccezione. Torino: Bollati Boringhieri, 2003.

AGIER, Michel. Managing the undesirables: refugee camps and humanitarian government. Cambridge: Polity Press, 2011.

AGIER, Michel. Un monde de camps. Paris: La Découverte, 2014.

AMOORE, Louise. Biometric borders: Governing mobilities in the war on terror. Political Geography, v. 25, 2006, p. 336-351.

\footnotetext{
22 SALTER, Mark B. When the exception becomes the rule: borders, sovereignty, and citizenship; AMOORE, Louise. Biometric borders: Governing mobilities in the war on terror; DOTY, Roxanne L. States of Exception on the Mexico-U.S. Border: Security, "Decisions," and Civilian Border Patrols; HOUTUM, Henk van, BOEDELTJE, Freerk. Europe's shame: Death at the borders of the EU.

${ }^{23}$ BARBERO, op. cit., p. 531.

${ }^{24}$ ISIN, op. cit.

${ }^{25}$ LENDARO, Annalisa. Investir la rue alors qu'on n'en a pas le droit. L'improbable mobilisation des demandeurs d'asile à Lampedusa (Italie).
} 
ANDERSON, Bridget. Mobilizing migrants, making citizens: migrant domestic workers as political agents. Ethnic and Racial Studies, v. 33, n. 1, 2010, p. 60-74.

ARENDT, Hannah. The origins of totalitarianism. New York, 1951.

BARBERO, Iker. Expanding acts of citizenship: the struggles of Sinpapeles Migrants. Social and Legal Studies, v. 4, n. 21, 2012, p. 529-547.

BROPHY, Susan D. Lawless sovereignty: challenging the State of Exception. Social and Legal Studies, v. 18, n. 2, 2009, p. 199-220.

CAMPESI, Giuseppe. La detenzione amministrativa degli stranieri. Roma: Carocci, 2015.

CUTTITTA, Paolo. Lo spettacolo della frontiera. Milano: Mimesis, 2012.

DOTY, Roxanne L. States of Exception on the Mexico-U.S. Border: Security, "Decisions," and Civilian Border Patrols. International Political Sociology, v. 1, 2007, p. 113-137.

ELLERMANN, Antje. Undocumented migrants and Resistance in the Liberal State. Politics and Society, v. 38, n. 3, 2010, p. 408-429.

FANON, Frantz. The wretched of the earth. Grove Press, 1965.

GIUGNI, Marco; PASSY, Florence. Migrant mobilization between political institutions and citizenship regimes: A comparison of France and Switzerland. European journal of political research, v. 43, n. 1, 2004, p. 51-82.

HOUTUM, Henk van; BOEDELTJE, Freerk. Europe's shame: Death at the borders of the EU. Antipode, v. 41 n. 2, 2009, p. 226-230.

ISIN, Engin Fahri. Being political: genealogies of citizenship. Minneapolis: University of Minnesota Press, 2002.

ISRAËL, Liora. Usages militants du droit dans I'arène judiciaire: le cause lawyering. Droit et société, v. 3, n. 49, 2001, p. 793-824.

KOOPMANS, Ruud (ed.). Contested citizenship: Immigration and cultural diversity in Europe. Minnesota: University of Minnesota Press, 2005.

LACLAU, Ernesto. Emancipazione/i. Salerno: Orthotes Edizioni, 2012.

LENDARO, Annalisa. Investir la rue alors qu'on n'en a pas le droit. L'improbable mobilisation des demandeurs d'asile à Lampedusa (Italie). Sociologie et sociétés, v. XLVII, n. 1, 2015, p. 123-146.

MCGREGOR, JoAnn. Contestations and consequences of deportability: hunger strikes and the political agency of non-citizens. Citizenship Studies, v. 15, n. 5, 2011, p. 597-611.

OLIVERI, Federico. Migrants as activist citizens in Italy: understanding the new cycle of struggles. Citizenship studies, v. 16, n. 5-6, 2012, p. 793-806.

OPEN ACCES NOW. La face cachée des camps d'étranger-e-s en Europe. 2014. Disponibile su: <http://www.migreurop.org/IMG/pdf/ facecacheecampsetrangers-okweb.pdf $>$. 
RAJARAM, Prem Kumar; GRUNDY-WARR, Carl. The irregular migrant as homo sacer: Migration and detention in Australia, Malaysia, and Thailand. International Migration, v. 42, n. 1, 2004, p. 33-64.

SALTER, Mark B. When the exception becomes the rule: borders, sovereignty, and citizenship. Citizenship Studies, v. 12, n. 4, 2008, p. 365-380.

SOYSAL, Yasemin Nuhoğlu. Changing parameters of citizenship and claims-making: Organized Islam in European public spheres. Theory and society, v. 26, n. 4, 1997, p. 509-527.

TASSIN, Louise. Le mirage des hotspots. Nouveaux concepts et vieilles recettes à Lesbos et Lampedusa. Savoir/Agir, v. 36, n. 2, 2016, p. 39-45.

Articolo ricevuto il 25.07.2016

Accettato per la pubblicazione il 19.09.2016

Received for publication in July 25 2016

Accepted for publication in September 19 ${ }^{\text {th }}, 2016$

ISSN impresso 1980-8585

ISSN eletrônico 2237-9843

http://dx.doi.org/10.1590/1980-85852503880004807 